\title{
Terhart, Ewald
}

\section{Die Lehre in den Zeiten der Modularisierung}

Teichler, Ulrich [Hrsg.]; Tippelt, Rudolf [Hrsg.]: Hochschullandschaft im Wandel. Weinheim u.a. : Beltz 2005, S. 87-102. - (Zeitschrift für Pädagogik, Beiheft; 50)

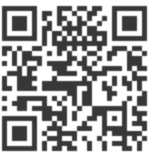

Quellenangabe/ Reference:

Terhart, Ewald: Die Lehre in den Zeiten der Modularisierung - In: Teichler, Ulrich [Hrsg.]; Tippelt, Rudolf [Hrsg.]: Hochschullandschaft im Wandel. Weinheim u.a. : Beltz 2005, S. 87-102 - URN:

urn:nbn:de:0111-opus-73918 - DOI: 10.25656/01:7391

https://nbn-resolving.org/urn:nbn:de:0111-opus-73918

https://doi.org/10.25656/01:7391

in Kooperation mit / in cooperation with:

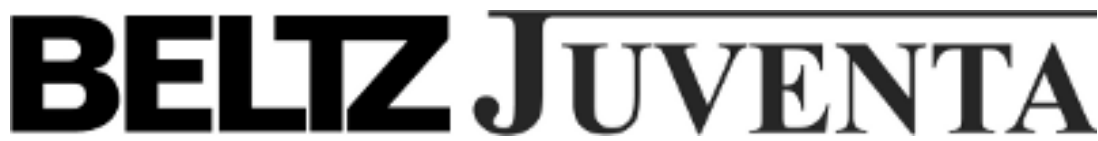

http://www.juventa.de

\section{Nutzungsbedingungen}

Gewährt wird ein nicht exklusives, nicht übertragbares, persönliches und beschränktes Recht auf Nutzung dieses Dokuments. Dieses Dokument ist ausschließlich für den persönlichen, nicht-kommerziellen Gebrauch bestimmt. Die Nutzung stellt keine Übertragung des Eigentumsrechts an diesem Dokument dar und gilt vorbehaltlich der folgenden Einschränkungen: Auf sämtlichen Kopien dieses Dokuments müssen alle Urheberrechtshinweise und sonstigen Hinweise auf gesetzlichen Schutz beibehalten werden. Sie dürfen dieses Dokument nicht in irgendeiner Weise abändern, noch dürfen Sie dieses Dokument für öffentliche oder kommerzielle Zwecke vervielfältigen, öffentlich ausstellen, aufführen, vertreiben oder anderweitig nutzen.

Mit der Verwendung dieses Dokuments erkennen Sie die Nutzungsbedingungen an.

\section{Terms of use}

We grant a non-exclusive, non-transferable, individual and limited right to using this document.

This document is solely intended for your personal, non-commercial use. Use of this document does not include any transfer of property rights and it is conditional to the following limitations: All of the copies of this documents must retain all copyright information and other information regarding legal protection. You are not allowed to alter this document in any way, to copy it for public or commercial purposes, to exhibit the document in public, to perform, distribute or otherwise use the document in public.

By using this particular document, you accept the above-stated conditions of use.

\section{Kontakt / Contact:}

\section{peDOCS}

DIPF | Leibniz-Institut für Bildungsforschung und Bildungsinformation Informationszentrum (IZ) Bildung

E-Mail: pedocs@dipf.de

Internet: www.pedocs.de

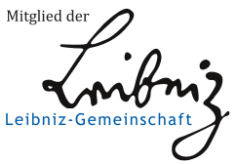


Zeitschrift für Pädagogik · 50. Beiheft

\section{Hochschullandschaft im Wandel}

Herausgegeben von Ulrich Teichler und Rudolf Tippelt

Beltz Verlag · Weinheim und Basel 
Die in der Zeitschrift veröffentlichten Beiträge sind urheberrechtlich geschützt. Alle Rechte, insbesondere das der Übersetzung in fremde Sprachen, vorbehalten. Kein Teil dieser Zeitschrift darf ohne schriftliche Genehmigung des Verlages in irgendeiner Form - durch Fotokopie, Mikrofilm oder ein anderes Verfahren reproduziert oder in eine von Maschinen, insbesondere Datenverarbeitungsanlagen, verwendbare Sprache übertragen werden. Auch die Rechte der Wiedergabe durch Vortrag, Funk- und Fernsehsendung, im Magnettonverfahren oder auf ähnlichem Wege bleiben vorbehalten. Fotokopien für den persönlichen oder sonstigen eigenen Gebrauch dürfen nur von einzelnen Beiträgen oder Teilen daraus als Einzelkopie hergestellt werden. Jede im Bereich eines gewerblichen Unternehmens hergestellte oder genützte Kopie dient gewerblichen Zwecken gem. \54 (2) UrhG und verpflichtet zur Gebührenzahlung an die VGWort, Abteilung Wissenschaft, Goethestr. 49, 80336 München, bei der die einzelnen Zahlungsmodalitäten zu erfragen sind.

(C) 2005 Beltz Verlag · Weinheim und Basel

Herstellung: Klaus Kaltenberg

Gesamtherstellung: Druckhaus »Thomas Müntzer«, Bad Langensalza

Printed in Germany

ISSN 0514-2717

Bestell-Nr. 41151 


\section{Inhaltsverzeichnis}

\section{Strukturelle Entwicklung und Steuerung des Hochschulwesens}

Ulrich Teichler

Quantitative und strukturelle Entwicklungen des Hochschulwesens

Hans Merkens

Zur Wettbewerbsfähigkeit des Hochschulsystems in Deutschland

Barbara M. Kehm/Ute Lanzendorf

Ein neues Governance-Regime für die Hochschulen mehr Markt und weniger Selbststeuerung?

David Phillips

Some Concerns in Higher Education in England: A Personal Note

\section{Wissenskulturen, Studienreform und Lehre}

Dirk Rustemeyer

Universitäre Wissenskulturen

Klaus-Peter Horn

Studienreform in der Erziehungswissenschaft -

Zwischen Pluralisierung/Diversifizierung und Zentrierung/Vereinheitlichung .........

Ewald Terhart

Die Lehre in den Zeiten der Modularisierung

Bernhard Schmidt/Rudolf Tippelt

Besser Lehren - Neues von der Hochschuldidaktik?

\section{Erziehungswissenschaft: Berufsübergang und Fächervergleich}

Cathleen Grunert/Ivo Züchner/Heinz-Hermann Krüger/Thomas Rauschenbach

Der Preis des Erfolgs? Hauptfach-Pädagogen und -Pädagoginnen im Beruf

Thomas Rauschenbach/Rudolf Tippelt/Horst Weishaupt/Ivo Züchner

Erziehungswissenschaft im Fächervergleich 


\section{Förderung des wissenschaftlichen Nachwuchses}

Jürgen Enders

Wissenschaftlicher Nachwuchs in Europa

Julia A.M. Delius/Nicola von Hammerstein

International Max Planck Research Schools:

Neue Wege der Graduiertenausbildung 170

\section{Evaluation und Ranking}

Carmen Lebherz/Cordula Mohr/Matthias Henning/Peter Sedlmeier

Wie brauchbar sind Hochschul-Rankings? Eine empirische Analyse

Hildegard Schaeper

Hochschulbildung und Schlüsselkompetenzen -

Der Beitrag der Hochschulforschung zur Evaluation der Qualifizierungs-

funktionen und -leistungen von Hochschulen 


\section{Ewald Terhart}

\section{Die Lehre in den Zeiten der Modularisierung}

Es gibt wohl kaum ein anderes Wort im Kontext der gegenwärtigen Umstellung der Studiengänge an Hochschulen, an das so viele Hoffnungen geknüpft sind und das zugleich so viel Verunsicherung und Ängste hervorruft wie „Modularisierung“. Wie andere, ähnliche Zentralworte in Politik und Gesellschaft hat auch „Modularisierung“ einen Signalcharakter angenommen. Ein solches Wort steht für einen komplexen Sinnzusammenhang, der bei der Verwendung des Wortes immer mittransportiert wird, aber eben gerade nicht mehr erläutert oder präzisiert werden muss. Zugleich ist ein solches Wort in einen normativen und emotionalen Bedeutungshof eingebettet, wobei die Richtung der Bewertungen und das Ausmaß der Emotionen individuell durchaus unterschiedlich sein können. Die Abkürzungs- und Signalfunktion solcher Worte kommt nur zustande, weil keine präzise und vereinheitlichte Bedeutung, keine genaue Definition o. Ä. vorhanden ist. Wird sie eingefordert und werden Erläuterungen gegeben, löst sich der Signalcharakter sofort auf. Auf Flaggen darf kein Text stehen.

So viel aber wissen mittlerweile alle Hochschulangehörige: „Module“ sind Elemente in Studiengängen, und „Modularisierung“ steht für den Prozess der Erarbeitung einer neuartigen Form der Strukturierung von Studiengängen, die dann eben aus „Modulen“ bestehen. Als Ergebnis dieses Prozesses ist der Studiengang „modularisiert“. Im Hochschulbereich sind mit dem Begriff „Modularisierung“ gegenwärtig im Wesentlichen folgende Assoziationen verbunden:

- stärkere Strukturierung der Lehrangebote und des Ablaufmusters im Studiengang, ggf. mit höheren Verbindlichkeiten für Lernende wie Lehrende;

- falls mehrere Disziplinen an einem Studiengang beteiligt sind: stärkere fachübergreifende Verzahnung der Angebote;

- Ablösung des Systems der Studienorganisation durch "Semesterwochenstunden“ und „Scheine“; Ende der herkömmlichen Form des Studiennachweises durch das „Belegen“;

- studienbegleitende Prüfungen, kontinuierlicher Erwerb von Leistungspunkten und Aufsummierung der Einzelleistungen zum Studienabschluss - ggf. ohne ein ,dramatisches' Abschlussexamen;

- Hoffnung auf Erleichterung des Wechsels zu anderen Universitäten im In- und Ausland;

- Änderung im Verhältnis von Erstausbildung, Berufslaufbahn und Weiterbildung; Möglichkeit des Zusammenstellens individueller Studienprofile;

- Pflicht zur Akkreditierung von neu entwickelten Studiengängen vor Aufnahme des Studienbetriebs; kontinuierliche Qualitätssicherung und Re-Akkreditierung. 
Diese Themen beschäftigen derzeit wohl jeden Fachbereich, jedes Institut an jeder Hochschule in Deutschland mehr oder weniger intensiv.

Ich möchte mich im Folgenden nicht mit allen Assoziationen und Facetten beschäftigen, die mit Modularisierung verbunden sind, und auch nicht die Frage erörtern, ob das Ganze sinnvoll ist und ob es die Ziele erreichen kann und wird, die es offiziell erreichen soll. Es geht auch nicht um die Einführung der Bachelor/Master-Struktur (vgl. dazu Keedy 1999; Oelrich 2001; Schwarz-Hahn/Rehburg 2004; Winter 2004; Horn 2005), denn systematisch gesehen ist Modularisierung hiervon unabhängig. Stattdessen geht es um Ereignisse und Erfahrungen, die mit dem konkreten Prozess der Erarbeitung von Modulen verbunden sind, um die konkreten, alltagspraktischen Konsequenzen, die dieser Prozess für die Organisation der Lehre in einem Institut oder Fachbereich sowie für das eigene Handeln innerhalb der Lehre hat. Aber auch die sehr grundsätzlichen Folgen dieser neuen Formen von Lehrorganisation für die Disziplin selbst bzw. für das Verhältnis der Disziplinvertreter zu dem Wissen ihrer Disziplin werden anzusprechen sein. Ich gehe dabei insbesondere auf die Situation in der universitären Lehrerbildung ein, die aufgrund ihrer über sehr viele Fächer und Fakultäten, verteilten' Struktur vielleicht nicht typisch ist für universitäre Studiengänge insgesamt, von der aber behauptet wird, dass gerade sie aufgrund dieser besonderen Lage auf Modularisierung angewiesen ist und von ihr profitieren kann. An ihr können also Glanz und Elend der Modularisierung recht gut verdeutlicht werden. Ich stütze mich dabei auf eigene, subjektive und insofern keinesfalls repräsentative Erfahrungen in Universitäten im Prozess der Modularisierung sowie bei der Bewältigung der praktischen Konsequenzen dieser neuen Formen von Lehre. Insofern kann man das Folgende auch als die Verarbeitung eines Selbstversuchs verstehen.

\section{Was heißt im Wortsinne „Modul“?}

Der Begriff Modul stammt ursprünglich aus dem Bereich der technischen Konstruktion. „Modul“ ist ein bestimmtes, abgegrenztes Element innerhalb einer komplexeren Struktur, z.B. ein Element innerhalb einer elektronischen Anlage. Dieses „Modul“ selbst kann in unterschiedlichen Anlagen eingesetzt werden, verrichtet aber der Sache nach immer den gleichen Dienst. So kann man ggf. aus bereits bestehenden Einzelmodulen durch neue Anordnung eine gänzlich neue Anlage erstellen. Im Maschinenbau und in der Autoindustrie, in der Bauindustrie sowie bei der Erstellung von Computerprogrammen werden „Module“ eingesetzt. Entscheidend ist jeweils, dass der Zweck des Moduls im Kontext der Gesamtstruktur sowie die Schnittstellen zwischen einem Modul und seinen Nachbarmodulen, mit denen es in direktem Austausch steht, genau definiert sind. Module können selbst wiederum aus einzelnen Elementen bestehen, und eine aus Modulen zusammengesetzte Anlage kann ein Modul innerhalb einer größeren Anlage bilden. Insofern hängt es im Grunde von der jeweils angelegten Perspektive bzw. der Grenzziehung ab, was man denn nun als ein ,Teil' und was als, Ganzes' betrachten will. 
So weit der Hintergrund in den Ingenieurwissenschaften. Innerhalb eines pädagogischen bzw. erziehungswissenschaftlichen Kontextes tauchten die Begriffe „Modul“ und „Modularisierung“ etwa seit Mitte der 1990er-Jahre keineswegs zuerst mit Blick auf die Lehre an wissenschaftlichen Hochschulen, sondern in der beruflichen Ausbildung (Erstausbildung und Weiterbildung) auf (Ziehm 1998; Leesemann 1998; Gonon 1998; Kloas 1999; Buschfeld 2000; Rützel 2002). Modularisierung steht hier für eine Form der Bereitstellung von Aus- und Weiterbildungsangeboten, die sehr stark zerlegt und auf individuelle Voraussetzungen zugeschnitten sind bzw. von den Interessenten zugeschnitten werden können. Während dies für die Organisation von Weiterbildung immer üblich war, weil man dort schon immer von sehr heterogenen Voraussetzungen, Motiven, Interessen auszugehen hatte, bedeutet Modularisierung von Erstausbildungen für Deutschland einen tiefgreifenden Wechsel. Traditionell gilt hierzulande nämlich eine enge Standardisierung von normierten Ausbildungswegen, die zu präzise definierten, aber gleichwohl komplexen Berufen führen, denen die Einzelnen dann ihr Leben lang nachgehen. Veränderungen in den Berufszuschnitten, in den Karrieremustern sowie in den vorgelagerten Bildungslaufbahnen, vor allem aber die rasante Beschleunigung aller dieser Veränderungen haben jedoch dazu geführt, dass diese standardisierte Formierung sich zugunsten einer schlussendlich hoch individualisierten, flexiblen und sehr risikoreichen Verknüpfung von Ausbildungserfahrungen, Berufschancen und tatsächlicher beruflicher Tätigkeit auflöst. Im Zuge dieser Entwicklung löst sich z.B. auch die Differenz zwischen Ersausbildung und Weiterbildung auf. Ehedem standardisierte Wege und garantierte Zugänge verändern sich in Richtung auf teils vorstrukturierte, teils individuell zusammenzustellende Menues von Bildungs-Modulen; Arbeiten, Lernen und Leben sind damit - dramatisch formuliert - vollständig und unablässig voneinander durchdrungen.

Auf der Ebene der höheren Bildung (Universität, Fachhochschule) verbindet sich die Karriere von „Modularisierung“ mit dem Bologna-Prozess, in dessen Rahmen eine europaweite Vereinheitlichung der Studienverläufe und -abschlüsse im Hochschulsystem erreicht werden soll; so ist zumindest die in Deutschland dominierende Lesart dieses Prozesses (zur Modularisierung im Hochschulsystem vgl. BLK 2001, 2002; HIS 2001; KMK/BMBF 2005). ${ }^{1}$ Die gerade skizzierten, auf Weiterbildung und Berufsbildung bezogenen Denkmodelle wurden gewissermaßen auf die höhere Bildung übertragen. Im Folgenden steht jedoch nicht das gesamte Bündel der Gründe für oder gegen „Bologna“ zur Debatte, sondern nur dessen im engeren Sinne curricularer, ausbildungsdidaktischer und prüfungsbezogener Teil: die Modularisierung - genauer, die Probleme und Konsequenzen, die im Rahmen der praktischen Erarbeitung und Umsetzung solcher Lehr-Strukturen auftreten.

1 Die Zeitschriften „Bildung und Erziehung“ (2005, Heft 2), „Journal für LehrerInnenbildung“ (2004, Heft 4) sowie „Forschung und Lehre“ (2005, Heft 2) beschäftigen sich ebenfalls mit „Modularisierung“. Ein erster Erfahrungsbericht aus der Lehrerbildung in Bielefeld findet sich bei Tillmann (2005). 


\section{Was bedeutet „,modularisieren“ - und wie macht man das?}

Der grundlegenden Idee nach verlangt Modularisierung, dass ein Studiengang als Prozess der schrittweisen Herausbildung von definierten Fähigkeiten („Kompetenzen“) betrachtet wird, wobei diese Fähigkeiten auf eine wissenschaftliche, in den allermeisten Fällen aber wohl eher auf eine außerwissenschaftliche berufliche Tätigkeit gerichtet sind. Ausgangspunkt ist insofern eine Liste derjenigen Kompetenzen, die Absolventen (und insofern dann: Berufsanfänger) haben sollten. Dies ist das wohl wichtigste und zugleich gravierendste Element von Modularisierung: Jedes Modul muss zu einer benannten, genau umrissenen Kompetenz führen - wobei der Wert dieser Kompetenz sich an ihrem Beitrag zur Herausbildung des schlussendlich angestrebten Fähigkeitsbündels bemisst. Der Ausprägungsgrad dieser Kompetenzen darf ein Minimum nicht unterschreiten und weist nach oben hin aufsteigende Kompetenzstufen oder Leistungsniveaus auf. Aus dieser Kompetenzliste werden dann diejenigen Studienerfahrungen ,abgeleitet', die die Lernenden machen müssen, damit dann schließlich diese Kompetenzen auch vorliegen. Die Lernerfahrungen wiederum sind inhaltlich und methodisch zu benennen und in eine zielführende Sequenz zu bringen - wobei kontinuierlich Elemente der Selbst- und Fremdbewertung einzubauen sind. Die einzelnen Module, aus denen ein Ausbildungsgang zusammengesetzt ist, müssen - wie erwähnt - selbst bereits zu einer genau definierten Einzel-Kompetenz führen. Die für die Absolvierung der Module notwendigen Leistungen und Arbeitsbelastungen der Studierenden (gerechnet in Arbeitsstunden) müssen benannt sein. Das ECTS-System gibt hierfür den Rahmen ab (ECTS 2004; HRK 2004); schlussendlich schreibt es übrigens auch eine bestimmte Verteilung der zu vergebenden Noten auf die einzelnen Notenstufen vor. ${ }^{2}$ Aus der Erledi-

2 Dieser Aspekt wird nicht selten übersehen. Nach einer Information der HRK (2004) wird auch im ECTS-System die Note lokal vergeben, und zwar nach der deutschen Notenskala von 1 bis 5. Diese Note wird ergänzt durch eine ECTS-Note, die international kompatibel ist oder sein soll. Die Verteilung der ECTS-Noten soll folgendermaßen aussehen. Es gibt fünf Stufen für erfolgreich absolvierte Leistungen: Stufe A ist für die besten $10 \%$ reserviert, Stufe B für die nächsten $25 \%$, Stufe C für die nächsten $30 \%$, Stufe D für die nächsten $25 \%$, und Stufe E für die nächsten $10 \%$. Stufe FX bedeutet „Nicht bestanden - es sind Verbesserungen erforderlich, bevor die Leistungen anerkannt werden können“, und Stufe F bedeutet „Nicht bestanden - es sind erhebliche Verbesserungen erforderlich“. Eine Datenabschrift dokumentiert die Leistung jedes Studierenden durch Aufstellung der absolvierten Kurse, der erworbenen Credits sowie der erzielten Noten und möglicherweise ECTS-Noten. Zusätzlich heißt es in dem HRKPapier: „Die Angabe von Misserfolgsquoten in der Datenabschrift ist nicht obligatorisch“ (HRK 2004, S. 3). Das bedeutet, dass bei dokumentierten Benotungen nicht jeweils angegeben werden muss, aber angegeben werden kann, wie hoch der Anteil der Durchgefallenen ist. Vor diesem Hintergrund korrigiert die HRK ihre Empfehlung aus dem Jahre 2000, die eine feste Umrechnung von deutschen Noten und ECTS-Noten vorschlug. Das ist konsequent, denn die Benotungsgewohnheiten an den deutschen Universitäten und ihren Fachkulturen entsprechen nicht der Notenverteilungsvorschrift des ECTS. Insofern sollen nun zwei Noten gegeben werden: eine nach deutschem Muster und eine nach ECTS. Die Implikationen und Folgen dieses Vorgehens brauchen hier nicht herausgearbeitet werden - sie sollten allen Sachkundigen unmittelbar klar sein. 
gung aller Teilelemente und den Einzelkompetenzen in der teilweise vorgeschriebenen Reihenfolge ergibt sich dann die abschließende Gesamt-Kompetenz.

So weit in kurzer Skizze die Idee. In der gegenwärtigen Praxis basiert jeder dieser Schritte wohl eher auf Entscheidungen und Probehandeln als auf eindeutigen und detaillierten Vorschriften, verlässlichen Erkenntnissen oder eingeübten Prozeduren. In den Universitäten und Hochschulen ist der skizzierte Prozess derzeit in reiner Form wohl kaum zu organisieren. Denn für die allermeisten akademischen Berufe wird man weder eine konsensfähige und präzise Aufstellung der notwendigen Kompetenzen noch eine wirklich alle Beteiligten überzeugende, also gleichsam zwingende Ableitung der zum Erwerb dieser Kompetenzen notwendigen Lernerfahrungen und deren Anordnung erstellen können - vor allem nicht für solche Berufe, die auf ein breit gefächertes und gestaffeltes, in sich differentes Spektrum von Positionen, Handlungsfeldern und Handlungsverläufen vorbereiten, das gar nicht im Einzelnen zu antizipieren ist. Besonders schwierig ist dies in den geistes- und sozialwissenschaftlichen Fächern, die - mit Ausnahme z.B. des Lehrerstudiums - noch am allerwenigsten auf definierte Berufspositionen und Handlungsmuster vorbereiten. Es existiert also eine Unsicherheit hinsichtlich der Wissensbasis für die Konstruktion solcher Studiengänge. Hinzu kommt als klar limitierender äußerer Faktor, dass man die modularisierte Lehre mit demjenigen Personal erzeugen (können) muss, das vorhanden ist. Unausweichlich muss jede Revision an die gegebene Ausgangssituation anknüpfen. Das bedeutet: man muss eine Art Kompromisslinie zwischen den gegebenen Ausgangsbedingungen und dem vorgestellten Ziel bzw. der ,reinen Lehre' finden. Die gegebenen Ausgangsbedingungen werden mehr oder weniger stark in die angestrebte Richtung umgearbeitet; in der Praxis des Modularisierens sind vielfältige Zwischenschritte und Teillösungen unumgänglich. So werden etwa die zu vermittelnden Kompetenzen eher vage beschrieben, die in den Modulbeschreibungen zu leistenden Inhaltsbenennungen werden exemplarisch und großzügig durch eine offene Liste verdeutlicht, die bislang in den Vorlesungsverzeichnissen durch Zwischenüberschriften mehr oder weniger mühsam in thematische Blöcke gegliederten Veranstaltungen werden zu Modulen bzw. Modulbereichen erklärt etc.

Da jede der vorgeschriebenen Veranstaltungen nicht mehr einfach „belegt" werden kann, sondern jede Veranstaltung auch tatsächlich besucht und zertifiziert werden muss, führt die einfache Übertragung des alten Planungsprinzips der „Semesterwochenstunden (SWS) “ auf die neue Studienorganisation zu einer Explosion der Lehrnachfrage, die wiederum eigentlich zu einer Erhöhung der Zahl der Veranstaltungen v.a. in den ausgewiesenen Pflichtbereichen führen muss. Da gegenwärtig und absehbar jedoch keine Ausweitung der Lehrkapazität durch mehr Personal zu erwarten ist, sondern stattdessen eher mit dem Gegenteil gerechnet werden muss, erfordert Modularisierung eine genauere, nachfrageadäquatere Verteilung der Art und Menge der angebotenen Veranstaltungen als bislang. Bei weitergehender Strukturierung wird eine im Kollegium verabredete einheitliche Grobplanung der einzelnen Veranstaltungen unausweichlich, denn in einem Modul (und seinen Elementen) soll schließlich das enthalten sein, was seine Bezeichnung verspricht. Ebenso werden über das jeweils zu planende Semester hinausgehende längerfristige Verabredungen zu treffen sein. Schließlich sind gemeinsame 
Standards für die notwendig werdende Zertifizierung und Benotung von Studienleistungen zwischen den Lehrenden abzusprechen. Diese Aufgabe wurde schon immer und wird gegenwärtig in den einzelnen universitären Einheiten durchaus gesehen. Ihre Erledigung wird aber früher wie heute gerne vertagt.

\section{Was geschieht, während man modularisiert?}

Die Modularisierung zu organisieren ist schon schwierig - sie tatsächlich durchzuführen noch schwieriger. Wer ist dafür zuständig? Wer hat die Federführung? Wer ist an der Erarbeitung beteiligt, und wer entscheidet über die Annahme? Wer hat die Kompetenz, die Planung Wirklichkeit werden zu lassen, auch dann, wenn Probleme, Widerstände und Verweigerungen zu verzeichnen sind? Die akademischen Gremien arbeiten erfahrungsgemäß sehr langsam und neigen zu formalen bzw. Verfahrensvorschlägen - im Prozess der Modularisierung muss man jedoch inhaltlich werden und in gewisser Weise auch Wertungen vornehmen zu Fragen wie: Für welche Felder und Aufgaben bilden wir Absolventen aus? Was sind Fähigkeiten, die unsere Absolventen in diesen Feldern benötigen? Was aus unserem vorhandenen Inhalts- und insofern dann auch: Personaltableau ist denn nun wie wichtig für welche Kompetenz - und was ist weniger wichtig? Und die herauszubildenden Kompetenzen sollen ja die berufsbezogenen Kompetenzen der Absolventen sein. Damit ist eine Positionsbestimmung zum systematisch schwierigen Verhältnis von Disziplin und Profession ein kontinuierlich mitschwingendes Grundthema.

Solche Diskussionen erfolgen in den zuständigen Arbeitsgruppen und Gremien faktisch und zu Recht immer mit ständigem Seitenblick auf die je persönlichen Qualifikationen und Ausrichtungen des vorhandenen Personals. Dieses ist aber noch unter anderen Prämissen und Philosophien zusammengesetzt worden - den Zufallsfaktor einmal nicht gerechnet. Allein deshalb bereits ist es schwierig, wenn nicht unmöglich, eine allein aus antizipierten Berufsfeldanforderungen abgeleitete Modulstruktur aufzubauen. Was geschieht mit denjenigen, die in einer so aufgebauten Struktur mit ihren Schwerpunkten nicht vertreten sind, nicht sein können - oder nicht sein wollen? Was geschieht, wenn das System zwar eingeführt wird, einzelne Lehrende oder Gruppen von Lehrenden das ganze Verfahren rundheraus ablehnen und weder ihre Veranstaltungen noch ihre Leistungsnachweise etc. ändern? Die Gelehrtenrepublik ist auf solche Situationen nicht wirklich vorbereitet. Faktisch kann und wird es natürlich - aufgrund von Modularisierung - an keinem Standort zu einer kurzfristigen Personalanpassung durch Personalerneuerung kommen; lediglich bei der Neubesetzung von Stellen kann man ggf. notwendige Umdefinitionen in Richtung auf die Modulstruktur vornehmen. Bei der Beantragung der (Wieder-)Besetzung von frei gewordenen Stellen können allerdings Fachbereiche und/oder Universitätsleitungen in der Tat einen mehr oder weniger starken Druck ausüben. Dies alles bedeutet: Man muss eine Modulstruktur aufbauen, die den neuen Anforderungen genügt und den Berufsbezug der Studiengänge stärkt, die es aber zugleich erlaubt, das vorhandene Personal insgesamt und mit seinen Schwerpunkten grosso modo in diese Struktur einzubinden. 
Ein grundsätzliches Problem zentriert sich um die Frage, wie viele der von den Studierenden zu erbringenden Leistungen eigentlich in welcher Weise zu Stande kommen bzw. ermittelt und bestätigt werden sollen. Welche Arbeitszeit/Arbeitsbelastung wird für welche Formen angesetzt? Werden alle zu erwerbenden Leistungspunkte an den Besuch von Veranstaltungen geknüpft, führt dies, wie erwähnt, in den bislang eher schwach strukturierten Studiengängen zu einer Vervielfachung der Lehrnachfrage. Insofern ist es klug, einen Teil der Leistungspunkte auch ohne direkten Bezug zum Besuch einer Lehrveranstaltung erwerbbar zu machen, wobei neue, geeignete Formen der Leistungserbringung und -überprüfung gefunden werden müssen. Oder aber man beschließt, nicht alle SWS in Leistungspunkte umzurechnen, wodurch der Studienumfang unter Umständen nominell reduziert wird. Ebenso besteht die Möglichkeit, Elemente der (reduziert verbleibenden) Abschlussprüfung mit Leistungspunkten zu versehen, sie also gewissermaßen zu Teilen des Studiums zu machen. Werden neben dem für alle Studierenden verbindlichen Kerncurriculum mehrere Wahlpflichtmöglichkeiten eröffnet, müssen diese allesamt hinreichend stark angeboten werden - auch wenn es womöglich nur wenige Studierende gibt, die sich für diese oder jene Wahlmöglichkeit entscheiden.

Sehr schwierig wird es, wenn mehrere Disziplinen an einem Studiengang beteiligt sind, wie dies typischerweise für Lehramtsstudiengänge insgesamt sowie speziell für deren erziehungswissenschaftliche Anteile gilt („Begleitstudium“ neben den Studium der Unterrichtsfächer und ihrer Fachdidaktik). Hier liefern die Disziplinen Erziehungswissenschaft, Psychologie, Soziologie, Politologie, Philosophie etc. einzelne Elemente hinzu. Die Strukturierung innerhalb dieses Studienelementes - am besten sogar: innerhalb der Module dieses Studienelementes - soll aber gerade nicht disziplinär, sondern problemoder besser: kompetenzbezogen sein. Für die beteiligten Fächer sind diese Angebote in der Lehrerbildung aber häufig Serviceleistungen. Entsprechend werden sie behandelt. Die Höhe des Abstimmungsbedarfs steht in umgekehrtem Verhältnis zur Abstimmungsbereitschaft. ${ }^{3}$ In der Lehrerbildung wird dies noch dadurch verschärft, dass die staatlicherseits erlassenen Prüfungsordnungen sowie die vor Ort befindlichen staatlichen Prüfungsämter eine limitierende und zensierende Funktion ausüben und dies auch weiterhin mit einigen guten (und einigen schlechten) Gründen tun wollen - andererseits aber nicht über wirklich durchgreifende administrative Mittel in Richtung Universität verfügen bzw. diese aus mancherlei Gründen nicht anwenden.

Schließlich ist bei Studiengängen, an denen mehrere Disziplinen beteiligt sind, gegenwärtig zu beobachten, dass (mangels einheitlicher Vorgaben oder in Unkenntnis der Vorgaben oder aus Eigenwille heraus) Disziplinen z.T. eigene Punktesysteme etc. entwickeln, was dazu führen kann, dass in einem Studiengang verschiedene Tarife gelten bzw. Studierende in ein und demselben Seminar für gleiche Leistungsformen unterschiedlich

3 An sehr vielen Standorten sind seit einigen Jahren Zentren für Lehrerbildung als zentrale wissenschaftliche Einrichtungen gegründet worden, die fakultätsübergreifend die Belange der Lehrerbildung vertreten, koordinieren und weiterentwickeln sollen (vgl. dazu Blömeke 2002; Terhart 2005). Zur Diskussion über das Verhältnis von Universität und Lehrerbildung generell vgl. von Prondczynsky (1998); Roth (1999); Blömeke (2003); Tenorth (2004). 
viele Punkte bekommen. Dies weist auf ein grundsätzliches Problem hin: Trotz einer anders lautenden Planung scheint es so zu sein, dass in den Bundesländern, zwischen den Standorten eines Bundeslandes und sogar zwischen Fakultäten ein und derselben Universität die vorhandenen bundes- und landesbezogenen Rahmenbestimmungen zur Reorganisation der Studienstrukturen im Sinne der Modularisierung sehr unterschiedlich ausgelegt werden. Dies ist eine Folge der Tatsache, dass der Bologna-Prozess einerseits (in Deutschland) de facto zentral verordnet ist (oder doch zumindest von interessierter Seite so behandelt wird) und auch entsprechende Rahmenbestimmungen existieren, andererseits aber im Zuge der ebenfalls zum Bolognaprozess gehörenden Autonomisierung der Hochschulen auf engem Raum differente Systeme entstehen können. Und dabei ist doch gerade Internationalisierung und höhere räumliche Mobilität das Ziel! Die Abstimmung den Akkreditierungsagenturen zu überlassen setzt gewissermaßen zu spät an; außerdem ist dies angesichts der Menge der zu akkreditierenden Studiengänge in einem zeitnahen Prozess gar nicht möglich. Und was geschieht eigentlich mit den Einrichtungen und Instituten, deren Konzepte für modularisierte Studiengänge trotz mehrfacher Anläufe endgültig nicht akkreditiert werden - und alle Alt-Studierenden zwar abgearbeitet, die Lehrenden aber noch sehr jung sind?

Insgesamt führt die Erarbeitung einer modularisierten Studienstruktur dazu, das Institute, Fachbereiche, Fakultäten sich plötzlich sehr intensiv über die Lehre, ihre Struktur und Inhalte sowie auch über den Zweck der Lehre - d.h. die Art der bei Studierenden anzubahnenden Kompetenz - auseinandersetzen. Das ist sicherlich als positiv zu bewerten. Miteinander sprechen ist aber immer auch riskant: Schon bei den allereinfachsten Fragen - Wie haben wir es bisher gemacht? - werden große Unterschiede zwischen den Lehrenden deutlich. Bei den Antworten auf gewichtigere Fragen werden die Unterschiede noch größer: Was sollen die Studierenden am Ende können? Zu welchem Zweck sollten sie dies können? Wie wollen wir das erreichen? Wie wollen wir feststellen, inwieweit sie dies können? Und schließlich ganz grundsätzlich: Was ist eigentlich der spezifische Charakter akademischer Studien bzw. Berufsausbildungen im Unterschied $\mathrm{zu}$ anderen Ausbildungsebenen und -zielen? Oder gibt es diese Unterschiede gar nicht? Viele dieser Themen waren in den Hochschulen unterschwellig schon immer drängend und kontrovers - sie brauchten aber nicht expliziert, institutionalisiert und für das eigene Handeln folgenreich erörtert zu werden. Die akademische Tradition und der Sozialisationsprozess als Wissenschaftler sorgten vielfach für ein frag- und diskussionsloses Einschwingen der Neulinge und Dazukommenden in die Üblichkeiten. In Zeiten der Modularisierung ist dies so nicht mehr möglich: Die bisherigen Gewohnheiten werden sich auflösen. An ihre Stelle treten neue.

\section{Womit muss man rechnen, nachdem man modularisiert hat?}

Der entscheidende Punkt zur Umsetzung der modularisierten Struktur ist die Zusammensetzung des Lehrangebots für ein Semester bzw. für mehrere Semester. Leider ist in der Vergangenheit diesem absolut entscheidenden Punkt zu wenig Sorgfalt gewidmet 
worden - man ahnt, warum. Nicht selten reichten die Lehrenden (z.T. in den engeren Arbeitsbereichen vorbesprochen) ihre Anmeldungen im Sekretariat ein, eine Gesamtliste wurde erstellt, und dann gewann das zuständige Gremium (das von den Einreichenden besetzt ist) gerne den Eindruck, dass - mit ein paar Verschiebungen hier und ein paar Bitten an Kollegen dort - das Ganze doch eigentlich sehr gut aussähe und den Studiengangsanforderungen entspräche. Die Studenten, die sich dann zu Beginn des Semesters ihren Wochenplan zusammenstellen wollten, sahen das dann nicht selten anders. Eine solche Form der Bildung des Lehrangebots ist im Zeitalter der Modularisierung nicht mehr möglich. Es muss eine genaue Einpassung der eingereichten Angebote in die Modulstruktur erfolgen; man wird für Teile des individuellen Angebots vom Prinzip der ,freien 'Einreichung von Veranstaltungen wegkommen müssen. Das Veranstaltungsangebot wird umgekehrt in großen Teilen thematisch vorstrukturiert werden müssen, und die Lehrenden ordnen sich Themen, Veranstaltungen etc. zu bzw. werden oder sind bereits zugeordnet. Dabei wird nicht in die genauen Inhalte und Methoden der Veranstaltung des einzelnen Lehrenden hineinregiert werden, aber der Rahmen und die Themenstruktur werden einheitlicher sein. Auf diesem Wege wird die notwendige Zahl von Veranstaltungen, insbesondere von Pflichtveranstaltungen, angeboten werden können. Vor allem aber wird es keine semesterweise, zufällig' zustande kommenden individuellen und institutionellen Lehrangebote mehr geben können, sondern stattdessen eine mittel- und längerfristige Planung der Lehrangebote über mehrere Semester hinweg. Nur auf diese Weise kann man erreichen, dass der Studiengang überhaupt im Rahmen der vorgesehenen Zeiten studierbar ist. Diese Planung schließt auch das Bemühen um eine sinnvolle Verteilung der Lehrveranstaltungstermine über die Woche ein. Wer erst jetzt die Freiheit von Forschung und Lehre ins Spiel bringt, muss sich fragen lassen, ob er ernsthaft meint, dass diese im Kern darin besteht, seine wöchentlichen Seminarzeiten frei wählen zu können.

Noch einmal muss ich auf einen Punkt zurück kommen, an dem alles hängt: In den Geistes- und Sozialwissenschaften gab und gibt es insbesondere, wenn diese Veranstaltungen für die Lehramtsstudiengänge relevant sind, eine Überfüllungssituation. Natürlich nicht in allen Veranstaltungen - aber doch in vielen. Dies gilt auch schon unter der Bedingung, dass viele Studierende zahlreiche Veranstaltungen lediglich zu „belegen“ haben - also nicht wirklich dort auftauchen und nur in wenigen Veranstaltungen tatsächlich „Scheine“ erwerben müssen. Anders: Bereits unter der Bedingung, dass nicht alle Studierenden wirklich alles machten, was sie zu machen hatten, platzte das System an manche Stellen aus den Nähten. Wenn nun die modularisierte Struktur so ausgelegt ist, dass alle Punkte nur durch den kontinuierlichen Besuch sämtlicher vorgeschriebener Veranstaltungen erworben werden können, explodiert das System. Mit Personalzuwachs ist aber absehbar nicht zu rechen. Die nahe liegendste Möglichkeit dies aufzufangen besteht darin, den Erwerb von Leistungspunkten vom Besuch von Veranstaltungen z.T. zu entkoppeln und neue Formen der Überprüfung der außerhalb von Seminaren erbrachten Leistungen zu finden. Es sollten auch nicht allzu viele Pflichtelemente für alle Studierende definiert werden: hat man dort nicht genug Personal bzw. Veranstaltun- 
gen, entstehen sofort ,Flaschenhälse ${ }^{`}$ und Studienzeitverzögerungen. Ebenso müssen angesichts dieser Lage zwingend mehr Vorlesungen durchgeführt werden, in denen dann auch möglichst viele anspruchsvolle Leistungen erbracht und also viele Leistungspunkte erworben werden können. Ohne (Groß-)Vorlesungen mit Klausuren in Studiengängen mit hohen Studierendenzahlen ist das System nicht umzusetzen. Gibt es hinreichend viele Vorlesungen, können dann die Seminare wiederum allmählich eine arbeitsfähige Größe erhalten.

Die Überlegungen zum Auffangen der Explosion der Lehrnachfrage führen schließlich vor ein strukturelles, sehr grundsätzliches Problem, das tatsächlich besorgniserregend ist: Es könnte sein, dass Modularisierung von Studiengängen so lange machbar ist und positive Effekte erzielt, solange die Studierendenzahl (und vielleicht auch die Zahl der einzubindenden Lehrenden und Fächer) nicht eine kritische Grenze überschreitet. Auf der Seite der Lehrenden wie der Lernenden werden ab einer gewissen Größe die Abstimmungsprobleme jedoch immer größer - und letztlich praktisch unlösbar. Dies wäre vielleicht sogar auch dann noch der Fall, wenn man auf der Seite der Lehrenden bzw. in den Fachbereichen klare und schnelle Möglichkeiten hätte, die notwendige Lehrstruktur zwangsweise herzustellen. Wird die Zahl der von Modularisierung ,betroffenen' Studierenden zu groß, ist das System vielleicht ebenfalls nur noch beherrschbar, wenn jedem Studierenden in jedem Semester sein Stundenplan vorgegeben wird. Das würde bedeuten, dass man bei großen Zahlen für Lehrende wie Lernende ein völlig verschultes, Zeiten und Inhalte einschließendes Zwangssystem einführen müsste. Das würde universitäre Lehre, ja: Universität unmöglich machen; an ihre Stelle träte eine (im doppelten Sinne) „Schein“-Institution. Für die Lehrerbildung, die an den größeren Universitätsstandorten der Republik jeweils Tausende von Studierende betrifft, die quer über zahlreiche Fakultäten hinweg stattfindet, und für die die Universität - selbst wenn sie wollte - nicht wirklich von einer Stelle aus alle ,Anbieter ${ }^{\prime}$ und ,Nachfrager ${ }^{\prime}$ zwangsorganisieren kann, ist das dann doppelt unmöglich, wenn diese paradoxe Formel einmal erlaubt ist. Angesichts solcher struktureller Unmöglichkeiten verfährt die Universität wie alle Organisationen in ähnlicher Lage: man praktiziert Schein-Anpassung, d.h., man erarbeitet Verfahren und Pläne, erfüllt Auflagen etc. in formaler Weise, kontrolliert aber nicht wirklich, ob die Praxis dies alles auch umsetzt, überlässt die Probleme des Alltags den einzelnen Lehreinheiten und Lehrenden - und am Ende den Studierenden selbst.

\section{Wie reagieren Studierende und Kollegen?}

Natürlich unterschiedlich! Die Reaktion hängt davon ab, welchen Stellenwert man im konkreten eigenen Energiehaushalt der akademischen Lehre gibt, wie man den vergangenen und aktuellen Lehrbetrieb bewertet, welche Erfahrungen man bislang gesammelt hat - und wie weit man noch von der Pensionierung entfernt ist. Dies ist alles gut nachvollziehbar und in Rechnung zu stellen. Meine Erfahrung hat gezeigt, und viele Kollegen, die wie ich im Laufe ihres Berufslebens jemals die Funktion des ,Beauftragten für die Erstellung des Veranstaltungsverzeichnisses‘ (o.̈̈.) inne hatten, haben bestätigt, dass 
schon immer ein gewisser Teil der Lehrenden noch nie wusste oder auch nur wissen wollte, wie die Studiengänge im Einzelnen aufgebaut sind, in die ihre Lehrveranstaltungen eingeordnet sind. Aufgrund der allfälligen Änderungen etc. ist dies in der Tat manchmal schwer zu durchschauen. Ein anderer Teil passt seine Angebote an die neue Struktur an, versteht das System der Leistungspunkte sofort und setzt die Sache um. Das mittlere, sehr breite Feld wählt den Weg der langsamen allmählichen, z.T. nur mimetischen Anpassung an die neuen Verhältnisse. Neue Veranstaltungstitel sind schnell formuliert, man stellt Klausuren, man rückt ab vom System der Studentenreferate, mit denen ganze Semester gefüllt werden etc. Man erprobt sich an neuen methodischen Formen, man lässt Klausuren schreiben, entwickelt neue Prüfungsformen, hat vielleicht eine gewisse Durchfallquote - und macht dann Colloquien für die Durchgefallenen etc.

Skeptische Kollegen machen vielfach die Modularisierung für die auftretenden Probleme verantwortlich. Dem ist jedoch entgegenzuhalten, dass durch Modularisierung zu einem sehr großen Teil lediglich diejenigen Probleme deutlich werden, die immer schon da waren, aber ängstlich-großzügig beschwiegen wurden. In diesem Zusammenhang gibt es eine Art von Folklore, die etwa so zusammengefasst werden kann: In irgendeinem nicht näher präzisierten Früher gab es noch die Universität im eigentlichen Sinne mit herausragenden akademischen Lehrern und eifrigen Studenten, die in faszinierenden Vorlesungen und kleinen Seminaren saßen und an der Sache orientiert emsig arbeiteten. (Angereichert wird dieses Szenario dann unweigerlich mit Erzählungen aus der eigenen Studentenzeit). Nur diese Freiheit sei akademisch und erzeuge Qualität. Die Massenuniversität habe dies alles zerstört. Nunmehr seien - bislang glücklicherweise bei Weiterexistenz der Freiheit aufseiten der Lehrenden - die Studiengänge verschult, die Seminare überfüllt und die Studierenden vielfach inkompetent und/oder desinteressiert. Entweder hätte man die Studierendenzahl nicht steigern dürfen, oder parallel zum Steigen der Studierendenzahlen auch die Professorenzahl analog erhöhen müssen. Nun müsse man halt warten, bis alle Uninteressierten das überfüllten Seminar zwei oder drei Wochen nach Semesterstart verlassen hätten. Die wirklich Interessierten blieben; jetzt könne es losgehen. Eine solche Argumentation verklärt nicht nur die (eigene) Vergangenheit, sondern führt dazu, dass man gewissermaßen Studienorganisation durch die Kombination von Raumgröße und Frustration betreibt - und einen großen Teil der Studierenden durch unübersichtliche Seminarlandschaften irrlichtern lässt, in ein $\mathrm{Zu}$ falls- bzw. strategisches Minimalstudium treibt oder gleich ganz aus dem Studium vertreibt.

Studierende, die als neu Eingeschriebene unmittelbar auf die neuen, modularisierten Verhältnisse treffen, informieren sich in aller Regel sehr rasch und können sich dann in dem System bewegen. Natürlich kennen sie ähnliches aus dem Kurssystem der Oberstufe und verhalten sich strategisch. Das ist nur rational. Studierende, die bislang in den alten Strukturen studiert haben, können natürlich unter diesen Bedingungen ihr Studium auch zu Ende führen. Manche bedauern, andere beneiden die ,Modularisierten؛ Die Gleichzeitigkeit von ,alter' und ,neuer' Welt führt dazu, dass man vielfach beide Studierendengenerationen in den Veranstaltungen hat und die Studienorganisation, die Leistungsnachweise etc. in beiden Varianten erklären muss. Die Studierenden der ,alten` 
Welt beurteilen die Anforderungsmuster, denen die Kommilitonen in der ,neuen 'Welt ausgesetzt sind, durchweg ambivalent: An sich sei das Neue ja besser, denn jetzt würde das Studium endlich ernst genommen; zugleich aber seien sie froh, dass sie selbst nicht mehr davon betroffen seien ...

\section{Wie verändert Modularisierung die eigene Lehre in Programm und Praxis?}

Ich habe diesen Prozess der Umstellung auf modularisierte Strukturen und die Umsetzung dieser Struktur in der Praxis an zwei Universitäten erlebt, mitgestaltet und jeweils die Konsequenzen unmittelbar erfahren. Im Folgenden geht es mir um die eigenen LehrErfahrungen in modularisierten Verhältnissen sowie um einige Konsequenzen.

- Zunächst einmal wird eine bestimmte Tendenz, die schon seit Jahren deutlicher geworden ist, noch verstärkt: Die Lehre und ihre Inhalte lösen sich sehr stark von den eigenen Forschungsthemen und -aufgaben ab. Aufgrund des mehr oder weniger sanften Drucks in Richtung auf die Eingliederung der eigenen Lehre in die Pflichtstruktur sind große Übersichtsvorlesungen und wiederkehrende Standardseminare durchzuführen. Auch in diese fließt selbstverständlich die eigene Forschungserfahrung ein, aber Forschung selbst kann man dann nur noch im verbleibenden - früher hätte man gesagt - Oberseminar erörtern. Diese Seminare sind dann gewissermaßen reserviert für sehr gute Examenskandidaten und für die dritte Ebene der BolognaArchitektur: die Promotionsstudien. Andererseits muss man sich aber fragen, ob dies nicht ,früher' bereits auch so war. Das Auseinanderdriften von Forschung und (wachsenden Teilen der) Lehre ist nicht zuallererst ein bolognaiser Effekt, sondern Folge des langanhaltenden Spezialisierungsprozesses wissenschaftlicher Forschung in allen Disziplinen.

- Traditionell wurden „Scheine“ durch Referate, Hausarbeiten, Klausuren, kleinere Leistungsnachweise auch Protokolle, Kurzberichte etc. erworben. Aber nicht alle Seminarteilnehmer mussten "Scheine“ machen; viele nahmen einfach teil, ohne eine verabredete schriftliche Leistung erbringen zu müssen. Jetzt erbringen alle Leistungen - und alle diese Leistungen müssen zertifiziert werden. Da manche dieser Leistungen in Zwischenprüfungs- und Examensnoten einfließen, müssen sie unter Examensbedingungen zustande kommen und bewertet werden. Das erfordert den Aufbau eines entsprechenden Apparates; dies insbesondere dann, wenn man sehr große Vorlesungen mit Hunderten von Teilnehmern hat (Anmeldungen, Abmeldungen, Prüfungen, Bewertungsprozess, Ausgabe der Scheine, Quittung der Ausgabe, Archivierung etc.).

- Bei den großen Veranstaltungen (Vorlesungen) wird die vermehrte Durchführung von Klausuren unvermeidlich. Dies war in der Erziehungswissenschaft zumindest lange Zeit nicht die Regel; in den anderen großen Universitätsdisziplinen, die ebenfalls seit Jahrzehnten große Massen zu bewältigen haben, war und ist das übliche Praxis. Für die Erziehungswissenschaft, die bei eingerechneten Verpflichtungen in 
der Lehrerbildung hinsichtlich der Studierenden mittlerweile die zweit- oder drittgrößte Universitätsdisziplin ist (vgl. Rauschenbach/Züchner 2000, S. 39, FN 6), muss sich diese Tradition erst noch herausbilden. Sofern sich ein solches Massenfach wie die Erziehungswissenschaft darauf kaprizieren würde, seine Lehre nur noch an denen zu orientieren, die sich für die Sache wirklich und tief interessieren, wären viele Seminare vermutlich wie leergefegt. Man sollte sich - insbesondere beim Blick auf die erziehungswissenschaftlichen Studien in den Lehramtsstudiengängen - nicht der Illusion hingeben, dass die Seminare so voll sind, weil sich so viele Studierende für genau diese Themen interessieren. Das war schon in den vor-modularisierten Zeiten nicht so, und heute, wenn man Studiengänge voll ,ausmodularisiert' und alle Punkte nur in Verbindung mit Seminaren und Anwesenheitspflicht gemacht werden könne, ist es vermutlich noch weniger der Fall. In anderen Disziplinen ist dies auch so - nur macht sich dort niemand Sorgen darüber, wenn die Studierenden sich ,nicht wirklich 'für die Inhalte interessieren: Die Disziplin definiert schlicht, was gewusst werden muss. Wer das dann nicht weiß und auch nicht wissen will oder anderes wissen will, bekommt nicht den Abschluss. Niemand hat ihn gezwungen, den Abschluss anzustreben. Vielleicht hätte er etwas anderes machen sollen.

Dies bringt mich zu einem Punkt, an dem die bisherigen Überlegungen zum Modularisieren in die eigene Disziplin zurückführen.

\section{Wie wirkt die Modularisierung auf die Haltung zur eigenen Wissenschaft zurück?}

Modularisierung, vor allem aber der mit ihr gegebene Zwang zur Herstellung einer definierten Verbindung zwischen Studienerfahrung und daraus resultierenden Kompetenzen stellt viele Disziplinen, ja letztlich die Universität in ihrem Selbstverständnis auf eine harte Probe. Grundsätzlich geht es um das Verhältnis von Wissenschaft und Beruf, von Disziplinen und Professionen, von einzelnen Teilbereichen einer Disziplin in ihrer Bedeutung für die Erzeugung von beruflich relevanten Fähigkeiten. Was kann Universität hier leisten - und was nicht? Wo ergibt sich die Notwendigkeit der Änderung - was kann man beibehalten? In den verschiedenen akademischen Kulturen sieht dies sicherlich jeweils anders aus. In den erziehungswissenschaftlichen Studiengängen wird man sich nicht an präzise umrissenen Berufen orientieren können - die Kompetenzen werden eher breit anzulegen sein. Mit Blick auf die Lehrerbildung, die ja zunächst in der universitären Phase, dann durch den anschließenden Vorbereitungsdienst (Referendariat) auf einen definierten und hochspezialisierten Beruf vorbereitet (eine je individuelle Konstellation aus Lehramt und Fächerkombination), sind die Kompetenzen vielleicht noch eher zu umreißen (vgl. dazu neuerdings KMK 2004). Dabei kann für die Universität natürlich nicht der berufsfertige Lehrer das Ziel sein, sondern der möglichst gut auf den Vorbereitungsdienst vorbereitete Absolvent. 
Für die Konstruktion eines Studiengangs ist dann die Frage entscheidend, welches Wissen aus welcher Disziplin man für relevant und kompetenzerzeugend hält. Da für die Entstehung von Kompetenz für pädagogische Berufe die Bedeutung erziehungswissenschaftlichen Wissens für die Entstehung von Kompetenzen für pädagogisches Handeln (als Lehrer, Sozialpädagoge etc.) aber durchaus unklar ist und von den verschiedenen Richtungen der Erziehungswissenschaft darüber hinaus deutlich unterschiedlich eingeschätzt wird, stellt sich bei der konkreten Arbeit an der Modularisierung die Gretchenfrage, welche Bedeutung und welchen Wert man der eigenen Wissenschaft bzw. dem eigenen Schwerpunkt innerhalb dieser Wissenschaft eigentlich zuweisen will. Was traut man ihr zu - was nicht? Je weniger man vom Wert der eigenen Disziplin für auBerwissenschaftliche, berufliche Zwecke überzeugt ist, desto zurückhaltender wird man bei der Modularisierung sein, da es eigentlich keine zwingenden Gründe gibt, diese oder jene Inhalte in dieser oder jener Sequenz zu einem Studiengang zusammenzufügen. Die bei vielen Erziehungswissenschaftlern verbreitete, leicht skeptisch-ironische Haltung zur eigenen Zunft, zur eigenen Disziplin und zur kompetenzerzeugenden Wirksamkeit des erziehungswissenschaftlichen Wissens wird diese Haltung vielleicht noch unterstützen.

Weitere Verunsicherungen und Reflexionsanlässe kommen hinzu: Sich z.B. intensiv mit der Bewertung von Klausuren sowie den Studentenantworten auf Klausurfragen zu beschäftigen, und sich hierbei - aufgrund der hohen Zahlen (s.o.) - um ein gewisses $\mathrm{Maß}$ an Standardisierung zu bemühen, führt immer wieder vor die Frage, was denn nun eigentlich ,richtig' und ,falsch' ist bzw. bei stärker reflexionsorientierten Aufgabenstellungen: Was als eine gerade noch hinreichende oder aber als vollständig überzeugende Argumentation zu bewerten ist. In den Geistes- und Sozialwissenschaften gibt es die unterschiedlichsten Auffassungen und Lehrmeinungen zu den verschiedenen Themen und Problemen. Sich schlicht auf das wirklich Konsensfähige zu beziehen, würde das Niveau der Darstellung und der Klausurfragen sehr stark herunterdrücken. Wie stark kann man eine bestimmte Position oder Argumentation zur (allein) richtigen machen - und die anderen zu falschen? Hilfsweise achtet man auf die Qualität der Begründungen für diese oder jene Position; das ist dann schon eine komplexere Leistung und erfordert einen komplizierteren Bewertungsprozess. Zugleich sind Erziehungs- und Bildungsfragen vielfach weltanschaulich und normativ durchtränkt. Umgekehrt sind auf der Karte der streng sachbezogenen erziehungswissenschaftlichen Forschung die weißen Flecken größer als die erkundeten Gebiete. So entsteht bei Prüfungen und Bewertungen vielfach eine Situation, in der man nicht mehr nur den Inhalt der Ausführungen selbst der erfreut sich in vielen Teilen einer hohen Bandbreite des noch Vertretbaren -, sondern sehr stark die Art der Argumentation etc. zu bewerten hat.

Trotz zahlloser Unwägbarkeiten, ungeklärter Probleme und auffälliger Paradoxien der Modularisierung halte ich es für ihren entscheidenden Vorteil, dass nunmehr alle beteiligten Individuen wie Instanzen in den Hochschulen gezwungen sind, der Lehre mehr Aufmerksamkeit zu widmen und sich selbst hinsichtlich ihrer individuellen Lehrtradition stärker zu reflektieren und zu kontrollieren. Dies müssen die für die Lehre verantwortlichen Gremien auf institutioneller Ebene tun; zugleich muss es jeder einzel- 
ne Lehrende individuell tun. Die ungeheuere Energie, die derzeit bundesweit darauf verwendet wird, lohnt nur deshalb, weil es gewissermaßen um die Fortsetzung bzw. Wiederankurbelung der Studienreform mit anderen Mitteln geht - wenngleich natürlich unter anderen gesellschaftlichen Voraussetzungen. Denn die herkömmliche und für Deutschland kennzeichnende institutionelle Formation von Wissenschaft, Forschung und Ausbildung für (akademische und nicht-akademische) Berufe stellt sich derzeit um. Die Hochschullandschaft in Deutschland erlebt gegenwärtig einen grundsätzlichen Gestaltwandel, der in seiner Bedeutsamkeit und Folgewirkung nur noch mit den humboldtschen Reformen zu vergleichen ist. Vielleicht ist dieser Wandel noch stärker als der von „1968“. In zehn bis fünfzehn Jahren wird in Universitäten und Fachhochschulen fast nichts mehr so sein wie es einmal war. Die Chancen der Modularisierung zu nutzen, ihre Risiken zu kontrollieren sowie vor allem: ihre tatsächlichen Ergebnisse zu erfassen und diese Erkenntnis in den kontinuierlichen und unabschließbaren Prozess der (Um-) Gestaltung der Hochschullandschaft einzubringen wird dabei von ganz entscheidender Bedeutung sein.

\section{Literatur}

BLK (2001): Modularisierung in Hochschulen. BLK-Fachtagung am 23. Mai 2001 in Hamburg. (Materialien zur Bildungsplanung und zur Forschungsförderung, Heft 98). Bonn.

BLK (2002): Modularisierung in Hochschulen. Handreichung zur Modularisierung und Einführung von Bachelor- und Masterstudiengängen. Erste Erfahrungen und Empfehlungen aus dem BLK-Programm „Modularisierung“. (Materialien zur Bildungsplanung und zur Forschungsförderung, Heft 101). Bonn.

Blömeke, S. (2002): Zentren für Lehrerbildung und ihr Beitrag zur Integration der Lehrerausbildungsphasen. In: Hinz, R./ Kiper, H./Mischke, W. (Hrsg.): Welche Zukunft hat die Lehrerausbildung in Niedersachsen? Hohengehren: Schneider, S. 254-265.

Blömeke, S. (2003): Universität und Lehrerausbildung. Bad Heilbrunn: Klinkhardt.

Buschfeld, D. (Hrsg.) (2000): Moderate Modularisierung - eine nationale und internationale Differenzierungsstrategie? Bielefeld: Bertelsmann.

ECTS (2004): ECTS Users' Guide. European Credit Transfer and Accumulation System and Diploma Supplement. Directorate-General for Education and Culture. Brüssel, August 2004.

Gonon, Ph. (1998): Modularisierung als reflexive Modernisierung. In: Berufliches Lernen im Wandel - Konsequenzen für die Lernorte? Nürnberg: Institut für Arbeitsmarkt- und Berufsforschung, S. 305-321.

HIS (2001): Neue Anforderungen an die Prüfungsverwaltung. Modularisierte Studiengänge und Diploma Supplement. Bericht zur Tagung des HIS-Kompetenzzentrums Prüfungsverwaltung. (HIS-Kurzinformation A/2001). Hannover: HIS.

Horn, K.-P. (2005): Studienreform in der Erziehungswissenschaft - Zwischen Pluralisierung/ Diversifizierung und Zentrierung/Vereinheitlichung. In: Teichler, U./Tippelt, R. (Hrsg.): Hochschullandschaft im Wandel. 50. Beiheft der Zeitschrift für Pädagogik. Weinheim: Beltz, S. 76-86.

HRK (2004): ECTS als System zur Anrechnung, Übertragung und Akkumulierung von Studienleistungen. Entschließung des 98. Senats vom 10.2.2004.

Keedy, J.L. (1999): In Stufen zum Ziel. Zur Einführung von Bachelor- und Master-Graden an deutschen Universitäten. Stuttgart: Raabe. 
KMK (2004): Standards für die Lehrerbildung: Bildungswissenschaften. Beschluss des Plenums vom 16.12.2004 und Bericht der Arbeitsgruppe. Bonn.

KMK/BMBF (2005): Realisierung der Ziele des Bologna-Prozesses. Nationaler Bericht 2004 für Deutschland von KMK und BMBF (unter Mitwirkung von HRK, DAAD, Akkreditierungsrat, fzs und Sozialpartnern). Bonn/Berlin.

Kloas, P.-W. (1997): Modularisierung in der beruflichen Bildung. Modebegriff, Streitthema oder konstruktiver Ansatz zur Lösung von Zukunftsproblemen? Berichte zur Beruflichen Bildung, 208. Bielefeld.

Leesemann, H.-D. (1998): Durch Modularisierung zum Dilettantismus. In: Der berufliche Bildungsweg 1998, H.3, S. 11-13.

Oelrich, G. (2001): Gestufte Studienstruktur in der Erziehungswissenschaft. Zur Einführung von neuen Studiengängen. In: Der pädagogische Blick 9, S. 165-182.

Prondczynsky, A.v. (1998): Universität und Lehrerbildung. In: Zeitschrift für Pädagogik 44, S. 6182.

Rauschenbach, Th./Züchner, I. (2000): Studierende. In: Otto, H.-U. u.a.: Datenreport Erziehungswissenschaft. Opladen: Leske + Budrich, S. 33-46.

Roth, R.A. (Hrsg.) (1999): The Role of the University in the Preparation of Teachers. London: Falmer.

Rützel, J. (2002): Modularisierung: Reformoption oder marktkonforme Anpassungsqualifizierung. In: Zeitschrift für Bildungsverwaltung 17, S. 23-46.

Schwarz-Hahn, St./Rehburg, M. (2004): Bachelor und Master in Deutschland. Empirische Befunde zur Studienstrukturreform. Münster: Waxmann.

Terhart, E. (2005): Zentren für Lehrerbildung: systematische Probleme, institutionelle Widersprüche, praktische Schwierigkeiten. In: Merkens, H. (Hrsg.): Lehrerbildung: Zentren für Lehrerbildung. Wiesbaden: VS Verlag für Sozialwissenschaften, S. 15-31.

Tenorth, H.-E. (2004): Reform der Lehrerbildung als Element der universitären Studienreform. In: Grimm, A. (Hrsg.): Die Zukunft der Lehrerbildung. Loccumer Protokolle 11/03. RehburgLoccum, S. 51-61.

Tillmann, K.-J. (2005): Vom Lehramt zum Bachelor. Alltagserfahrungen mit dem Bielefelder Modell. In: Bastian, J./Keuffer, J./Lehberger, R. (Hrsg.): Lehrerbildung in der Entwicklung. Das Bachelor-Master-System: Modell - Kritische Hinweise - Erfahrungen. Weinheim: Beltz, S. 2934.

Winter, M. (2004): Ausbildung zum Lehrberuf. Zur Diskussion über bestehende und neue Konzepte der Lehrerausbildung für Gymnasium bzw. Sekundarstufe II. (HoF-Arbeitsberichte 3’04). Wittenberg: Institut für Hochschulforschung an der Martin-Luther-Universität HalleWittenberg.

Ziehm, St. (1998): Berufskonzept und Modularisierung. Leitideen beruflicher Bildung in Deutschland, den USA und Großbritannien. Alsbach: Leuchtturm.

Anschrift des Autors:

Prof. Dr. Ewald Terhart, Westfälische Wilhelms-Universität Münster, Institut für Schulpädagogik und Allgemeine Didaktik, Bispinghof 5/6, 48143 Münster. 